\title{
Atomic fluorescence emitted from a corona discharge in helium above and below saturated vapour pressure ${ }^{\star}$
}

\author{
Nagham M. Shiltagh ${ }^{1,2}$, Luis G. Mendoza Luna ${ }^{1, a}$, Mark J. Watkins ${ }^{1}$, Stuart C. Thornton ${ }^{1}$, \\ and Klaus von Haeften ${ }^{1, b, c}$ \\ ${ }^{1}$ Department of Physics and Astronomy, University of Leicester, Leicester LE1 7RH, UK \\ ${ }^{2}$ Department of Physics, College of Science, University of Kerbala, Karbala, Iraq
}

Received 9 May 2017

Published online 16 January 2018

(C) The Author(s) 2018. This article is published with open access at Springerlink.com

\begin{abstract}
A new apparatus was constructed to investigate the visible and near infrared fluorescence spectroscopy of electronically excited helium over a wide range of pressures and temperatures, covering both the gaseous and liquid phases. To achieve sufficient throughput, increased sensitivity was established by employing a micro-discharge cell and a high performance lens system that allows for a large collection solid angle. With this set-up, several thousand spectra were recorded. The atomic $3 s^{1} S \rightarrow 2 p^{1} P$ and $3 s^{3} S \rightarrow 2 p^{3} P$ atomic transitions showed line shifts, spectral broadening and intensity changes that were dependent in magnitude on pressure, temperature and thermodynamic phase. While in the gas phase the lines showed little dependency on the discharge cell temperature, the opposite was observed for the liquid phase, suggesting that a significant number of atoms were solvated. Triplet lines were up to a factor of 50 times stronger in intensity than the singlet lines, depending on pressure. When taking the particle density into account, this effect was stronger in the gas phase than in the liquid phase of helium. This was attributed to the recombination of $\mathrm{He}_{2}{ }^{+}, \mathrm{He}_{3}{ }^{+}$and $\mathrm{He}_{4}{ }^{+}$with electrons, which is facilitated in the gas phase because of the significantly higher mobility.
\end{abstract}

\section{Introduction}

The electronically excited states of condensed helium have attracted considerable attention in the literature. Unlike the other condensed rare gases, the spectra of helium clusters, droplets and liquid helium spectra show discrete, atomic- and molecular-like features [1,2]. The microscopic foundations of these peculiarities have been the focus of considerable research activity over the past 50 years; much of this research has been devoted to identifying how the quantum nature of fluids affects electronically excited states [3-6]. For example, the highly quantum nature of liquid helium is reflected in the isotope and implicit particle density-dependence of the energies of the electronically excited states [2]. Also, the non-radiative decay following electronic excitation of liquid helium droplets shows a dependence on the isotopic constitution and,

\footnotetext{
${ }^{\star}$ Contribution to the Topical Issue "Dynamics of Molecular Systems (MOLEC 2016)", edited by Alberto Garcia-Vela, Luis Banares and Maria Luisa Senent.

a Present address: Departamento de Física, Universidad Autónoma Metropolitana Iztapalapa, Av. San Rafael Atlixco No. 186 Col. Vicentina, C.P. 09340 México D.F., Mexico.

b e-mail: klaus.von.haeften@rub.de

${ }^{c}$ Present address: Kanano GmbH, 89075 Ulm, Cäcilie-Auer Weg 7, Germany.
}

implicitly, whether the droplets are superfluid or just normal liquid [7].

From a more generic point of view, such studies are also important because liquid helium is one of the purest - if not the purest - of all substances that exist. At the temperature where helium becomes liquid, all foreign chemical impurities must inevitably freeze out. This is important because defects and impurities generally affect electronic structure and dynamics rather strongly. The exceptional purity of cryogenic helium has recently been exploited in studies of nucleation and growth of nanoparticles around ions in supercritical helium [8].

Furthermore, helium is an interesting test system for theory. The atoms have only two electrons, which greatly facilitates high level ab initio calculations of clusters with several helium atoms involved [9-15].

A variety of excitation methods have been reported, including radioactive particles [16-21], electron beams $[1,22-29]$, proton beams [30,31], discharges [8,32-37] and field emission $[38,39]$. Helium clusters and droplets have been excited with electrons [40-45] and with VUV radiation [2,7,11,46-57], including high harmonic generation [58-60]. More recently, intense VUV radiation from free electron laser light sources has also been employed [61,62].

In this paper, the fluorescence of excited helium atoms in gaseous and liquid helium has been investigated at cryogenic temperatures. A corona discharge has been 
employed to produce electronic excitation. Corona discharges operate over a wide range of pressures and densities, and can produce a rich fluorescence spectrum in liquid helium [34-36]. It is therefore possible to examine the excited levels as a function of pressure, temperature and thermodynamic phase. Recently, this technique was adopted for the study of helium excimers in gaseous and liquid helium. The excimers exhibited characteristic line shifts that were dependent on pressure, temperature, thermodynamic phase and whether the molecules were fully solvated and in thermal equilibrium with the liquid helium or located in hot gas bubbles within the liquid [37]. Both blue and red shifts were observed. These shifts depended on the thermodynamic phase. The analysis of the spectral features also revealed that molecules located in different environments contributed to the observed lineshapes, in that the observed spectral features could be superpositions of molecules in different sites.

Here, we report on atomic spectra that have been recorded between 0.1 and 5.6 bar and on isotherms between 3.8 and $5.0 \mathrm{~K}$. This range covers the gas and the liquid phases of helium as our goal was to explore solvation effects with specific regard to the thermodynamic phase. Our work adds to previously available data sets: Soley and Fitzsimmons reported lineshifts of atomic transitions between saturated vapour pressure (SVP) and 25 bar for temperatures between 1.4 and $2.0 \mathrm{~K}$. Both absorption and emission were investigated. All lines were blue-shifted when pressure was increased [29]. Li and Bonifaci investigated atomic spectra above the SVP in normal liquid helium [34-36,63]; our results are in agreement with these previously published results. However, we report new data at pressures and temperatures that have not been investigated before, including in the region the below the SVP. Depending on the temperature, a large part of the line profile in the region below the SVP is red-shifted. Our observations are important as a benchmark for theory [64-70] and will contribute to a better understanding of collisional processes, including in the atmosphere of the sun, and other stars and exoplanets [67-76]. Our studies are also relevant to an understanding of micro-solvation. The excited helium atoms under consideration are in $n=3$ Rydberg states. The $3 s$ states are spherically symmetric and form bubbles of up to $15 \AA$ in diameter in liquid helium because of the Pauli-repulsion between the excited electron and the $1 s^{2}$ electrons of the neighbouring ground state atoms. Such states have similarities with anions which recently have been observed in helium clusters $[77,78]$; theory confirms that these clusters appear to have a similar bubble-like solvation structure [77-81].

To make this advance a significant amount of spectra had to be recorded in a reasonable time. This required the construction of a spectrometer with a highly efficient optical system which greatly reduced the data acquisition time compared to instruments used in the state of the art literature. With the new set up we were able to record spectra in under $100 \mathrm{~ms}$ with very good signal to noise ratio. Also we demonstrated the viability of a new micro-corona discharge cell for excitation, enabling the investigation of superfluid helium in the future. A great deal of our paper is devoted to reveal the details this new experimental set-up.

\section{Method}

To investigate the effects of pressure, temperature and thermodynamic phase on the fluorescence of helium atoms in a wider range we designed and built a new apparatus, conceptually similar to that previously used by $\mathrm{Li}$ et al. [36], but with better performance in a number of areas. A requirement was that the cell could operate at high pressures and at cryogenic temperatures around $4 \mathrm{~K}$. Another objective was to measure a larger number of spectra in a given time per experimental run than possible with the apparatus used by Li and coworkers. To accomplish this, sensitivity had to be increased by several orders of magnitude. This was achieved by a newly designed lens system which was able to collect fluorescence light over a larger solid angle and delivered the light to the detector with significantly fewer loss in intensity.

For the cell, a cube-like design was chosen, providing faces for flanges for two high voltage electrodes, the helium gas supply and a window to couple out the fluorescence light. The approximately cubic cell had a side length of approximately $50 \mathrm{~mm}$, providing an internal volume of $4 \mathrm{ml}$. It was made of oxygen-free copper (OFHC) to improve performance at cryogenic temperatures. Pressure tests showed that it could withstand over 100 bar. A section drawing of the cell is shown in Figure 1.

The tip and the plane electrodes were mounted opposite each other on stainless steel flanges using Macor to establish the desired point-plane discharge configuration. The tip had been etched from a tungsten wire, having a radius of $250 \mathrm{~nm}$ as verified by scanning electron microscopy. The gap between the electrodes was 3-4 mm wide depending on the actual tip used and the thickness of the seals (which were made of indium). On the outside of the cell, the electrodes were connected to phosphor-bronze leads (insulated with polyimide) which were heatsunk on the first $(80 \mathrm{~K})$, second $(4 \mathrm{~K})$ and third cooling stages $(300 \mathrm{mK}-4 \mathrm{~K})$. The electrical insulation of the wires was found to be insufficient for the required high voltage (in the $10 \mathrm{kV}$ range). PTFE sleeving had to be introduced, which reduced the effect of the heat sinking.

The cell was mounted centrally to the "pot" of a closed-cycle Oxford Instruments Heliox AC-V He3 using a home-made adaptor plate. It turned out that the ${ }^{3} \mathrm{He}$ stage, which was connected to the pot, did not provide sufficient cooling power. Therefore, the pot was bridged with the second stage using copper braids. The temperature was measured using a calibrated Cernox resistor (CX-1030-CU-HT0.3L) mounted to the outside of the cell. The precision of the resistor was $\pm 3 \mathrm{mK}$. The temperature of the helium inside the cell was verified at SVP and it was found that it deviated by no more than $0.1 \mathrm{~K}$ on the Cernox sensor readout. The temperature was changed via an electric heater and control circuit, which was part of the Oxford Instrument cryostat assembly. A cross-sectional view of the cell attached to the cryostat is shown in Figure 2.

Helium was supplied through a stainless steel pipe (Swagelok part number SS-T1-S-020-6ME) of $1.6 \mathrm{~mm}$ in diameter. The pipe was heat-sunk on the first $(80 \mathrm{~K})$ and the second stage $(4 \mathrm{~K})$ and connected through a 


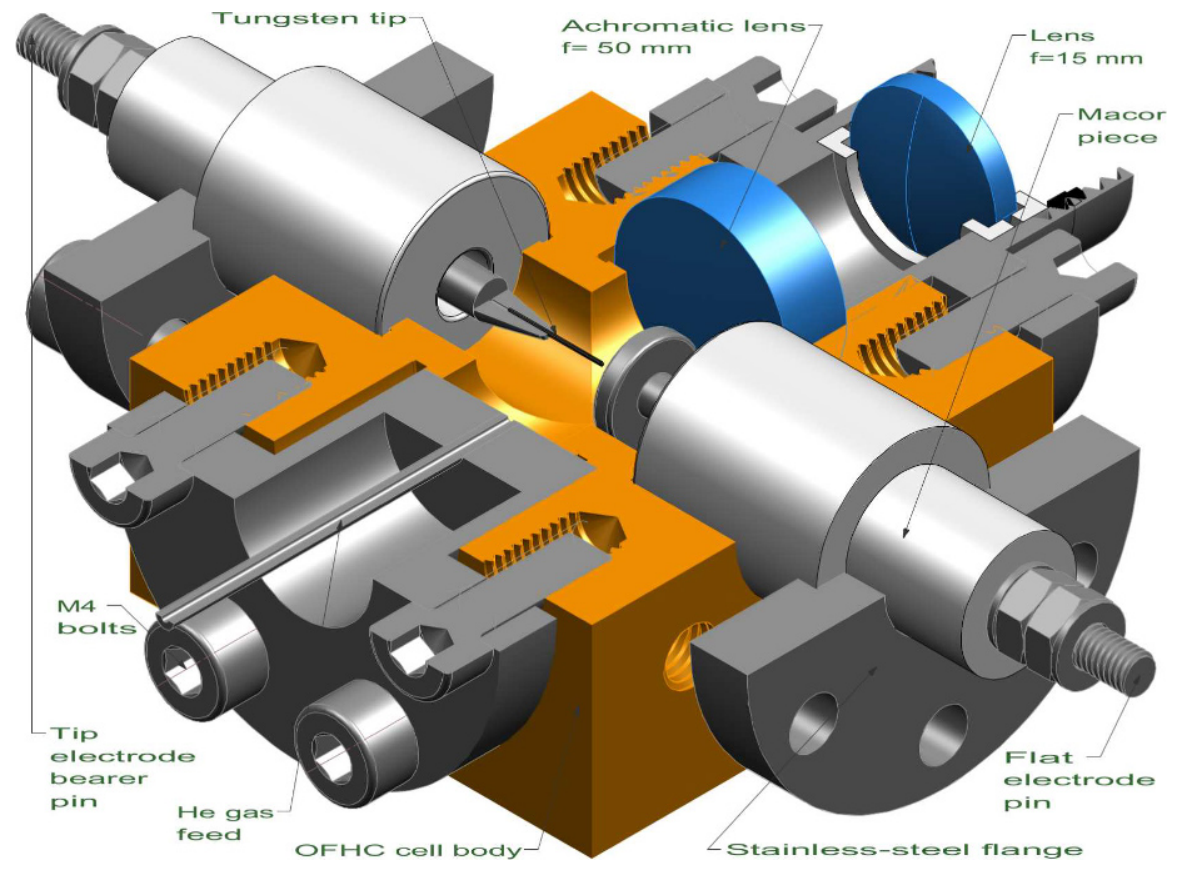

Fig. 1. Section view of high pressure cell.

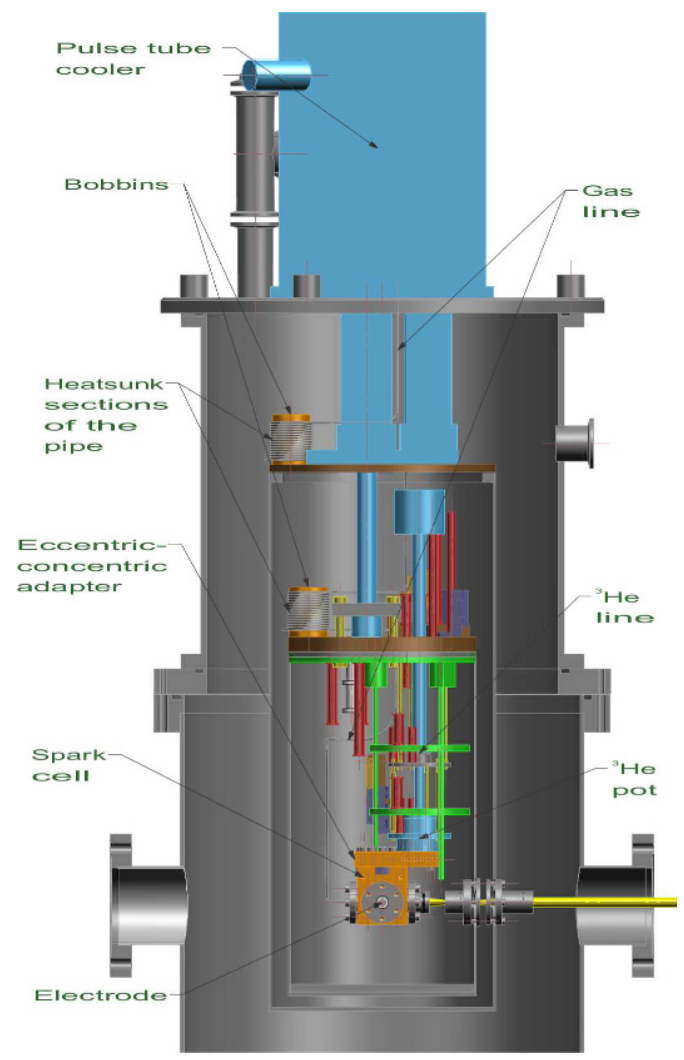

Fig. 2. Overview of the cryostat assembly showing the a cross section of the high pressure cell attached to the cryostat.

vacuum feedthrough with a gas-inlet system. The pressure was measured capacitively using a PXM409-USBH Omega transducer (0-100 bar absolute pressure). Helium of N6.0 research grade purity was supplied after repeatedly purging and evacuating the lines with a scroll pump.

After the initial evacuation of the cell, a glow discharge was ignited and reference spectra were recorded at room temperature. After starting the cooling-down process and reaching the base temperature and pressure $(3.2 \mathrm{~K}$ and about 0.150 bar), additional gas was introduced in increments into the cell. By doing this, the temperature of the cell increases temporarily but the refrigerator took it back to its base value, with the overall effect of increasing the pressure ever so slightly. The process was repeated until the point that small increments in the amount of gas in the gas line did not produce further increases in the pressure. This is the signature of the helium lying on the SVP curve of the phase diagram. To produce liquid helium, more helium needs to be added to the line until an increase in pressure is observed (after stabilisation at base temperature). When the pressure transducer indicates a pressure above the SVP (and $T$ is below $T_{c}$ ), liquid helium has been produced. When the helium was liquid, a corona discharge was ignited using high voltage in the region of $10 \mathrm{kV}$ which was provided by a Spellman MPS 10N10/24/DCC2 power supply. It should be noted that in the gas phase, the ignition voltage was significantly lower than in the liquid phase. We also note that on average the ignition voltage was in our set-up about twice as high than that of $\mathrm{Li}$ et al. who used a wider electrode separation [82].

Changing the pressure during operation was found to affect the discharge current and temperature of the cell. It was therefore not straightforward to record data along isotherms. Instead, we chose to record a large number of data points wherever we were able to establish stable conditions and select data points of interest from the large pool of data. Measurements at each point on the 


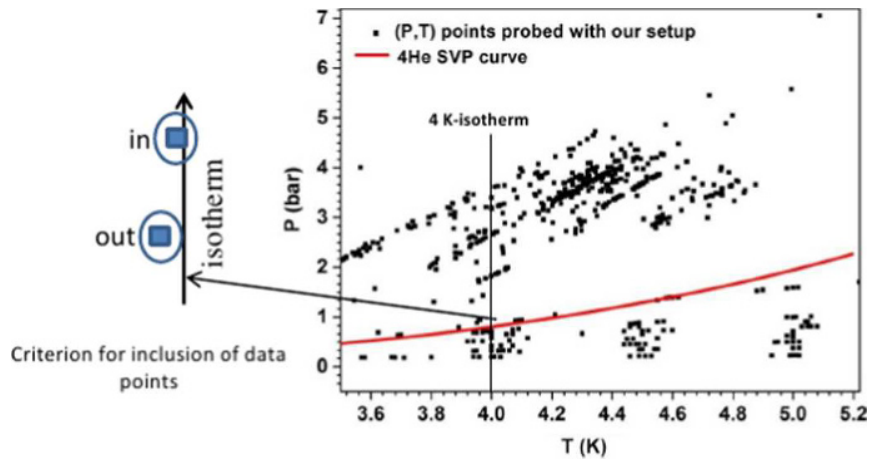

Fig. 3. A section of the $P T$ diagram for the gaseous and liquid phases of helium probed in the experiment. The saturated vapour pressure (SVP) curve of ${ }^{4} \mathrm{He}$ is marked as a red line, showing the difference in the phases of helium. Also, as an example, the selection criterion for inclusion of data as being on the isotherm is shown. The blue circles around the example data points are $0.1 \mathrm{~K}$ in diameter. Any point of data considered to be isothermic or isobaric is measured independently.

$P T$ diagram were repeated at least three times. The criterion to select data points on a specific isotherm was that the temperature deviated by not more than $0.1 \mathrm{~K}$ from the isotherm - our error in temperature - and that the independently measured spectra were consistent. The procedure for selecting data points is illustrated in Figure 3.

To collect the fluorescence light, a multiple lens system was used (see Fig. 4). An $f=50 \mathrm{~mm}$ achromatic lens (Edmund Optics Dwg. No. 47702INK) was placed $8 \mathrm{~mm}$ from the excitation zone. This lens simultaneously served as a sealing element. It was followed by a $f=15 \mathrm{~mm}$ convex lens (Thorlabs Part No. LB1092) which focused the fluorescence light to its focal point located approximately $75 \mathrm{~mm}$ away from the excitation zone and close to the radius of the radiation heat shield of the cryostat. This arrangement allowed a pinhole to be placed within the radiation shield to minimise heat impact from ambient radiation through the windows. The fluorescence light diverging from the pinhole was collimated by another $f=50 \mathrm{~mm}$ achromatic doublet, forming a parallel light beam that was directed via a vacuum view port onto a pair of metal-coated mirrors mounted on an optical table. An $f=150 \mathrm{~mm}$ achromatic doublet was used to focus the fluorescence light onto the slit of an Andor Technology Shamrock SR303i Czerny Turner spectrometer. Spectra were recorded with a Peltier-cooled $\left(-65^{\circ} \mathrm{C}\right) \mathrm{CCD}$ camera (Andor iDus DV420, CCD-12855). A $1200 \mathrm{~mm}^{-1}$ grating blazed at $500 \mathrm{~nm}$ was employed throughout the work presented in this paper. The resolution was $0.2 \mathrm{~nm}$. The spectral line profile of the instrument followed to a good approximation a Lorentzian function. This greatly facilitates deconvolution of the measured linewidths.

The acquisition time for each single spectrum was $10 \mathrm{~ms}$ for measurements in the gas phase and $100 \mathrm{~ms}$ in the liquid phase. The acquisition was repeated 20 times or 10 times for the gas and liquid phase, respectively, during which the spectra were accumulated and corrected for noise (cosmic event removal, etc.). This led to an effective exposure time, $t_{\text {eff }}=t_{\text {exp }} \times n_{\text {accu }}=0.2 \mathrm{~s}$ for the gas phase and $1 \mathrm{~s}$ for the liquid phase, respectively. Further details can be seen in [83].

\section{Results}

Spectra were selected from four isotherms, those at 3.8, $4.0,4.5$ and $5.0 \mathrm{~K}$. Here we concentrate on the $3 s^{3} S_{0} \rightarrow$ $2 p^{3} P_{0,1,2}$ and $3 s^{1} S_{0} \rightarrow 2 p^{1} P_{1}$ atomic transitions. Figure 5 shows the transition of singlet helium in the region of $728 \mathrm{~nm}$. Spectra have been recorded at various pressures, covering both the gas and the liquid phases. To better compare line profiles, the intensities have been normalised. For comparison, a glow discharge spectrum of atomic helium is also shown (dashed line).

At low pressure, the features consist essentially of only the atomic line. With increasing pressure, the line broadens and begins to develop a blue-shifted wing. With increasing pressure, this wing becomes a separate band that takes on an increasingly symmetric line profile.

The corresponding atomic triplet lines $\left(3 s^{3} S_{0} \rightarrow\right.$ $\left.2 p^{3} P_{0,1,2}\right)$ are shown in Figure 6. At low hydrostatic pressure, the atomic line is visible. This broadens with increasing pressure and develops a blue-shifted wing. With further increases in pressure, this wing becomes a symmetric band whose maximum shifts to shorter wavelengths with increasing pressure.

To assess lineshifts, line broadening and intensity, the spectral lines were fitted to a Lorentzian function. Lorentzian functions produced equally good fits to the data as more complicated functions, but had fewer free parameters than, for example, Voigt functions. Also, compared to other ways for the assessment of linewidth such as full width at half maximum Lorentzian functions have the benefit of a greatly simplified deconvolution process. The deviation between different methods was under $10 \%$. Details of this fitting procedure are given in our previous publication [55]. Figure 7 shows the lineshift obtained from this fitting procedure as a function of pressure for four isotherms. Correspondingly, Figure 8 shows the lineshift for the triplet line as a function of pressure for the same four isotherms.

The pressure dependence of the triplet $3 s^{3} S_{0} \rightarrow$ $2 p^{3} P_{0,1,2}$ transition and the associated lineshifts have been measured before by Soley and Fitzsimmons for pressures above SVP [29]. The lineshifts obtained from our measurements agree broadly with both these results and also with more recent measurements where corona discharge excitation has been employed [36,63]. To the best of our knowledge, lineshifts below SVP pressure have not been previously reported, and will be contrasted with values in the liquid phase.

Inspection of the pressure dependence of the lineshifts revealed regions where a linear dependence on pressure was apparent. These regions coincided with the gas and the liquid phases. We note that this coincidence cannot be taken for granted. The interaction between electronically excited helium atoms and ground state helium differs from the interaction between a helium ground state atom and another helium ground state atom. Also, many body 


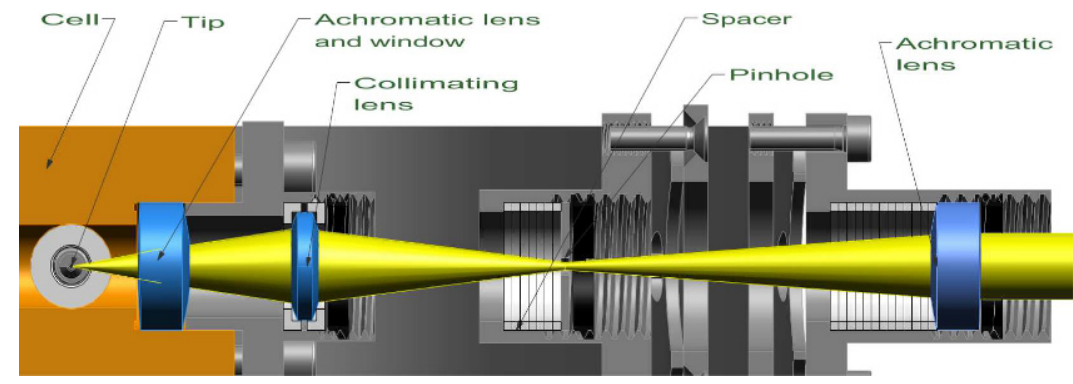

Fig. 4. Detailed schematic of the fluorescence light collection optical system.

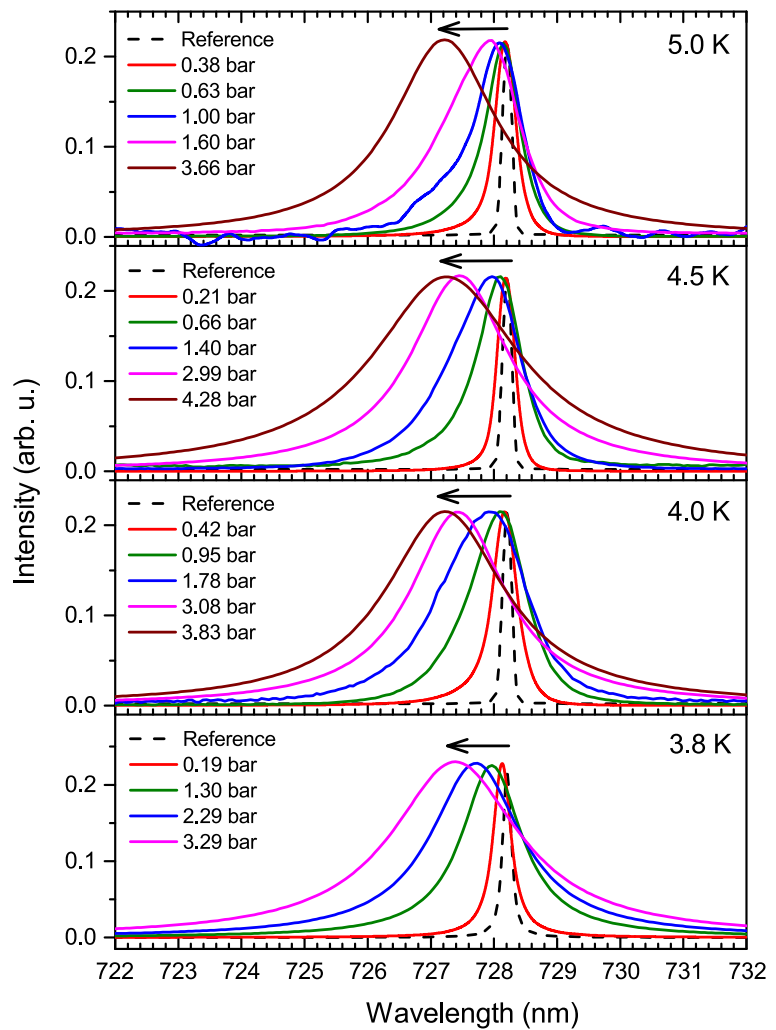

Fig. 5. Fluorescence spectra of gaseous and liquid helium excited by a corona discharge in the region of the atomic $3 s^{1} S_{0} \rightarrow 2 p^{1} P_{1}$ transition of singlet helium. All intensities have been normalised.

effects of these systems may differ. As a consequence, one cannot expect the phase transition line of a mixed system of excited helium atoms dissolved in ground-state atoms to coincide with the pressures and temperatures where the gas-liquid phase transition of pure helium occurs.

To establish trends, lineshifts were grouped together depending on whether the data points lie in the gas or in the liquid phase. The dashed lines in Figures 7 and 8 mark the boundaries of the crossover region where no data points were available, and hence the phase transition could not be explored in finer detail. The function $\Delta \lambda(p)=\frac{\partial \Delta \lambda}{\partial p} p+\Delta \lambda_{0}$ was fitted to the lineshifts. Lineshift coefficients, $\frac{\partial \Delta \lambda}{\partial p}$ and $\Delta \lambda_{0}$, were obtained for the gas and solvated phases. The coefficients are listed in

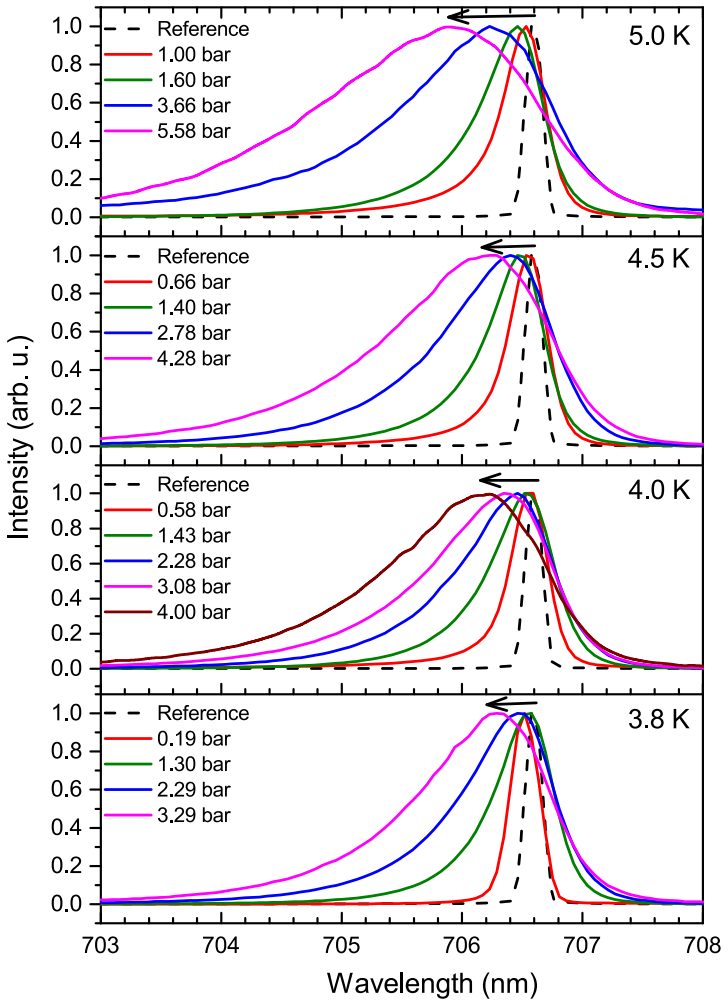

Fig. 6. Fluorescence spectra of gaseous and liquid helium in the region of the atomic triplet transition $3 s^{3} S_{0} \rightarrow 2 p^{3} P_{0,1,2}$. Intensities have been normalised.

Tables 1 and 2, with the exception of the $3.8 \mathrm{~K}$ isotherm where too few data points below SVP were available for reasonable analysis.

A similar procedure was undertaken to assess linewidths. The linewidths obtained from fitting the spectral data with Lorentzian functions are shown in Figures 9 and 10 for the 3.8, 4.0, 4.5 and $5.0 \mathrm{~K}$ isotherms. Similar to the treatments of lineshift, the data points were grouped together with respect to the thermodynamic phases and their apparent linear dependence on the pressure. A linear function was fitted to the data and linewidth coefficients were obtained. The resulting linear functions are shown in Figures 9 and 10 as straight solid lines, and the linewidth coefficients are listed in Tables 3 and 4 . Again, the dashed vertical lines mark the gap of data points where we have reason to suspect the crossover 


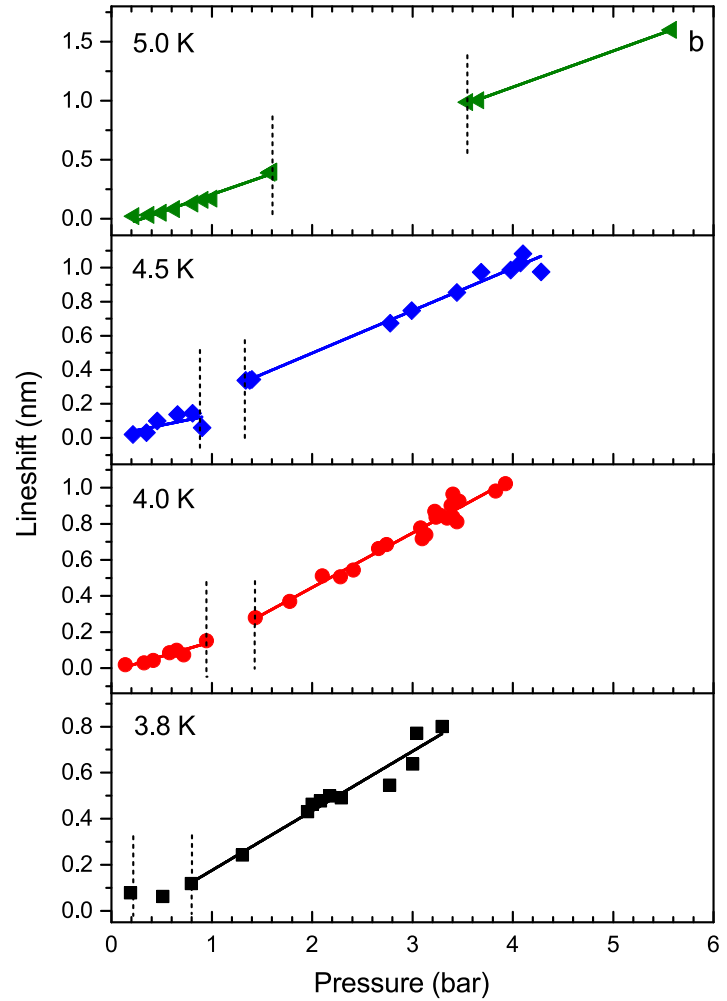

Fig. 7. Pressure dependence of the $3 s^{1} S_{0} \rightarrow 2 p^{1} P_{1}$ atomic singlet transition in the gas and liquid phases at 3.8, 4.0, 4.5 and $5.0 \mathrm{~K}$. The straight lines indicate regions that show linear dependence on pressure. The dashed lines show the region where the lineshift coefficient changes.

region between gas and liquid in assuming that for electronically excited helium the phase transition may not be exactly identical to that of pure helium. Our assumption is due to the changing slope of the linewidth coefficient trend lines and that this change is not always exactly on top of the SVP. These crossovers are identified in the same regions as with regard to the line shifts shown in Figures 7 and 8 . We note that for the $5 \mathrm{~K}$ isotherm the slope of the linear functions in the gas and the liquid phase are very similar. Also the data points are scarce. The grouping of data points is therefore slightly ambiguous. The data point at 1.6 bar could be grouped to the liquid phase which would produce an equally good fit. However, the crossover region would then lie between 1.0 and 1.6 bar which would be significantly below the phase transition of pure helium at 1.6 bar.

Also, the line intensities as obtained from fitting the spectral data with Lorentzian functions were examined. An apparent feature was that the intensity of the singlet lines was much lower than the intensity of the triplet lines (though this is not apparent from Figs. 5 and 6 ). The fluorescence intensity changes with pressure and temperature; an analysis without meaningful calibration is rather challenging. We have therefore divided the intensity of the triplet by that of the singlet lines prior to normalisation, which eliminates the effect of intensity changes due to external factors.

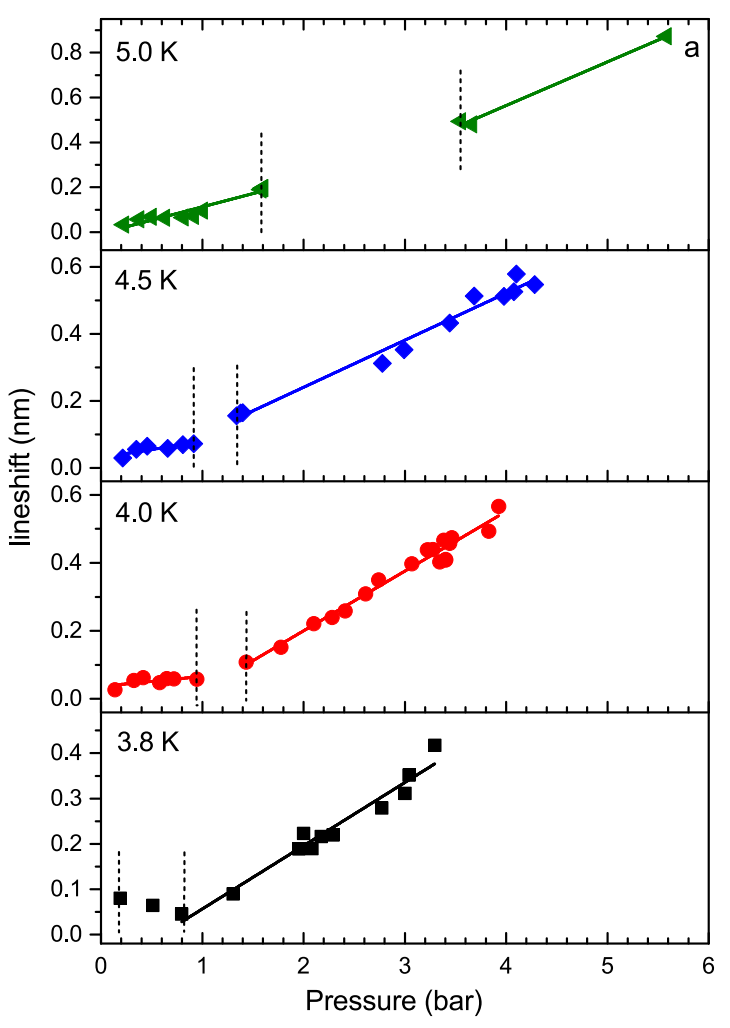

Fig. 8. Same as Figure 7 but for the triplet line $\left(3 s^{3} S_{0} \rightarrow\right.$ $\left.2 p^{3} P_{0,1,2}\right)$. Note the decreasing lineshift with pressure for $3.8 \mathrm{~K}$ in the gas phase.

\section{Discussion}

Corona discharges operating in a point-plane geometry in cryogenic helium create a confined region of excitation, producing a variety of localised electronically excited atomic and molecular transitions including the atomic transition which are at the focus of the present paper. The lineshift and linewidth observed for different pressures have been the topic of recent publications which show an inconclusive picture. Allard et al. [84] pointed out that the large linewidths reported by Li et al. [36] are incommensurate with the temperatures of the discharge cell and liquid helium. Also, the same authors stress that it is important to include in the analysis of lineshift interaction potentials over a large range due to the Rydberg nature of the states involved. Truncation at $R=16 \AA$ would be insufficient and lead to false results.

We add to these findings that our data shows significant differences between gas and liquid phase that rule out a quantitative analysis using the static or the impact approximation. These employ the density as the key parameter. The differences between gas and liquid phase unravelled in the present study shows that density alone cannot account for the observations.

Our recent publication shows $\mathrm{He}_{2}{ }^{*}$ molecular spectral features and population of high rotational quantum numbers equivalent to rotational temperatures of several hundred $\mathrm{K}$, which is clearly incommensurate with a thermal equilibrium with liquid helium [37]. When the 
Table 1. Lineshift coefficients for singlet atomic lines in the gas and the liquid phases.

\begin{tabular}{llll}
\hline$T[\mathrm{~K}]$ & & $\begin{array}{l}\text { Slope, } \frac{\partial \Delta \lambda}{\partial p} \\
{[\mathrm{~nm} / \mathrm{bar}]}\end{array}$ & $\begin{array}{l}\text { Intercept, } \Delta \lambda_{0} \\
{[\mathrm{~nm}]}\end{array}$ \\
\hline 4.0 & Gas & $0.16 \pm 0.03$ & $-0.02 \pm 0.02$ \\
4.5 & Gas & $0.23 \pm 0.04$ & $-0.03 \pm 0.02$ \\
5.0 & Gas & $0.30 \pm 0.02$ & $-0.10 \pm 0.02$ \\
& & & \\
3.8 & Liquid & $0.26 \pm 0.02$ & $-0.10 \pm 0.05$ \\
4.0 & Liquid & $0.30 \pm 0.01$ & $-0.16 \pm 0.04$ \\
4.5 & Liquid & $0.26 \pm 0.01$ & $-0.03 \pm 0.03$ \\
5.0 & Liquid & $0.31 \pm 0.01$ & $-0.11 \pm 0.03$ \\
\hline
\end{tabular}

Table 2. Lineshift coefficients for triplet atomic lines in the gas and the liquid phases.

\begin{tabular}{llll}
\hline$T[\mathrm{~K}]$ & & $\begin{array}{l}\text { Slope, } \frac{\partial \Delta \lambda}{\partial p} \\
{[\mathrm{~nm} / \mathrm{bar}]}\end{array}$ & $\begin{array}{l}\text { Intercept, } \Delta \lambda_{0} \\
{[\mathrm{~nm}]}\end{array}$ \\
\hline 4.0 & Gas & $0.03 \pm 0.02$ & $0.04 \pm 0.01$ \\
4.5 & Gas & $0.05 \pm 0.02$ & $0.03 \pm 0.01$ \\
5.0 & Gas & $0.12 \pm 0.01$ & $-0.01 \pm 0.01$ \\
& & & \\
3.8 & Liquid & $0.14 \pm 0.01$ & $-0.10 \pm 0.02$ \\
4.0 & Liquid & $0.18 \pm 0.01$ & $-0.15 \pm 0.02$ \\
4.5 & Liquid & $0.14 \pm 0.01$ & $-0.04 \pm 0.02$ \\
5.0 & Liquid & $0.20 \pm 0.02$ & $-0.21 \pm 0.10$ \\
\hline
\end{tabular}

hydrostatic pressure of liquid helium is increased, these molecules were found to cool at a rate of at least $10^{10}$ to $10^{11} \mathrm{~K} / \mathrm{s}$ via collisions with the liquid. At a pressure of a few bar practically all molecules end up in the lowest allowed rotational quantum state before they emit fluorescence. These findings suggest a picture where helium gas bubbles, driven by the corona discharge, co-exist within the body of the liquid helium. With increasing pressure, the volume available to the molecules in the bubbles decreases, equivalent to an increase of the concentration of helium in the gas bubbles [37]. However, we also found that the concentration of gas bubbles in the cell decreased compared to the concentration of fully solvated molecules, suggesting that increased pressure stimulated the solvation of the molecules. While this interpretation builds on previous findings for helium excimers, it is reasonable to assume a similar situation for helium atoms.

To test whether this assumption also holds for cryogenic gas, we compare the pressure dependence of the lineshift and linewidth coefficients and the triplet to singlet intensity ratios in the gas phase for the 3.8, 4.0, 4.5 and $5.0 \mathrm{~K}$ isotherms. All of these observables do not differ significantly between the different temperatures, with the exception of the $3.8 \mathrm{~K}$ isotherm. The most notable difference can be seen in the lineshift of the triplet atomic fluorescence, which decreases with increasing pressure in complete contrast to the trends observed at all other temperatures (Fig. 8).

In the liquid phase, lineshift and linewidth coefficients, and triplet to singlet intensity ratios, show a dependence on the cell temperature. The slope of the coefficients

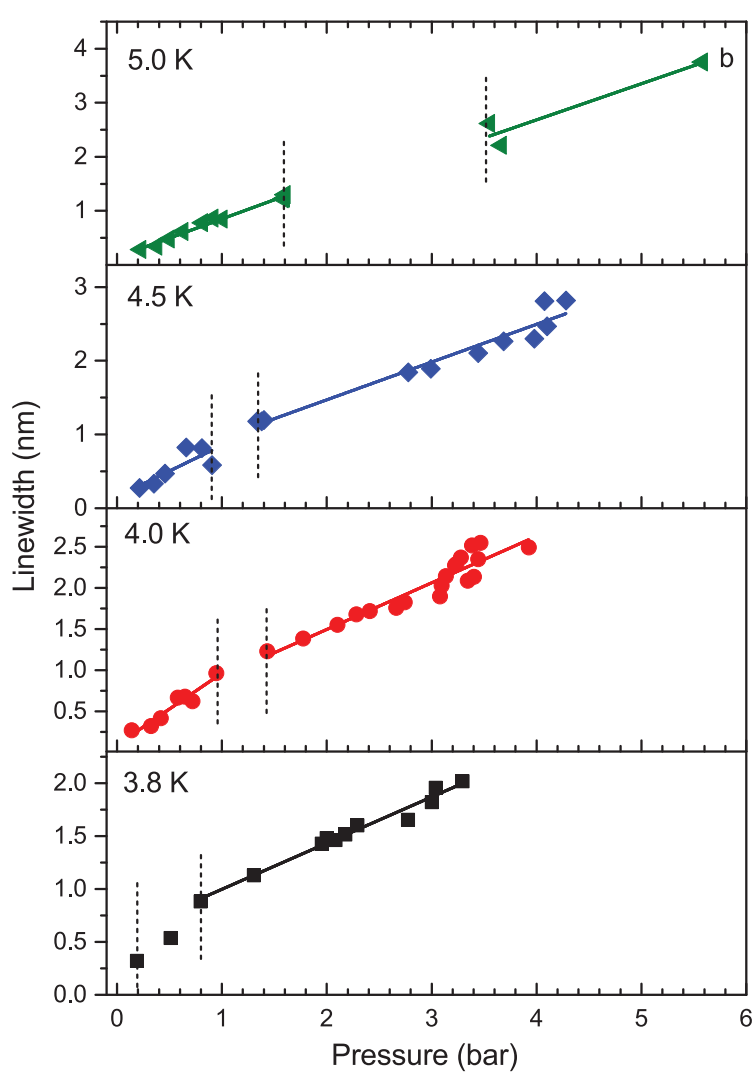

Fig. 9. Pressure dependence of the linewidth of the $3 s^{1} S_{0} \rightarrow$ $2 p^{1} P_{1}$ atomic singlet transition in the gas and liquid phases at $3.8,4.0,4.5$ and $5.0 \mathrm{~K}$. The straight lines indicate regions that show linear dependence on pressure. The dashed lines indicate the crossover regions. The unconvoluted, measured linewidths deduced from the fitting procedure are shown.

increases with temperature with the exception of the $4.5 \mathrm{~K}$ isotherm. The intercepts of the lineshift coefficients are negative, indicating that the atoms are in a different environment than the gas phase. Also, the magnitude of the intercept increases with temperature, showing a greater sensitivity to the environment than the gas phase.

In summary, there are many indicators to suggest atoms being solvated in liquid helium, or at least that a significant percentage of solvated atoms contribute to the spectral features. The possibility that atoms in gas bubbles contribute to the spectra cannot be excluded, however, as we do not have the tools to disentangle such a contribution.

The triplet-singlet intensity ratio shows an interesting dependency on both the pressure and temperature. In the gas phase, the intensity ratio of the two increases in a rather linear fashion. The slope of this increase is almost independent of the cell temperature, indicating that plasma properties in the corona discharge region dictate the mechanisms of the energy transfer processes. A linear increase suggests that the higher lying singlet states, $E\left(3 s^{1} S\right)=22.920 \mathrm{eV}$, undergo collisions with ground state helium atoms to form the lower-lying triplet states with an energy of $E\left(3 s^{3} S\right)=22.718 \mathrm{eV}$, which is $0.202 \mathrm{eV}$ lower than the singlet. 


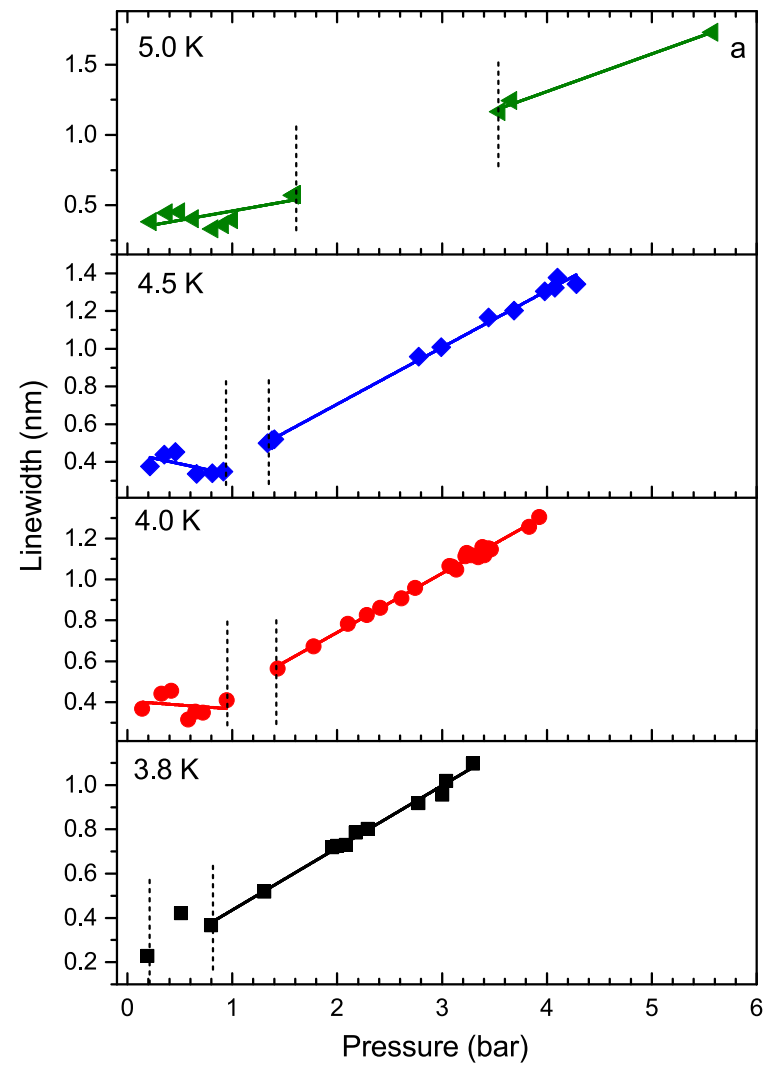

Fig. 10. Pressure dependence of the linewidth of the $3 s^{3} S_{0} \rightarrow$ $2 p^{3} P_{0,1,2}$ atomic triplet transition in the gas and liquid phases at $3.8,4.0,4.5$ and $5.0 \mathrm{~K}$. The straight lines indicate regions that show linear dependence on pressure. The dashed lines show crossover regions. The measured linewidths deduced from the fitting procedure are shown.

However, such a conversion is rather unlikely. Not only does bridging this energy difference of $202 \mathrm{meV}$ require rather high collisional temperatures, though this is in principle possible in a corona discharge plasma, but the Wigner-Spin rule would also be violated [85]. Singlettriplet transfers have been observed and investigated over many decades, including more recently in helium clusters and droplets that were electronically excited using monochromatic synchrotron radiation [50,53]. Wellenstein and Robertson showed that in a gas excited by a glow discharge, the Wigner-Spin rule was not violated and that associative ionisation, $\mathrm{He}^{*}+\mathrm{He} \rightarrow \mathrm{He}_{2}{ }^{+}+\mathrm{e}^{-}$followed by dissociative recombination, $\mathrm{He}_{2}{ }^{+}+\mathrm{e}^{-} \rightarrow \mathrm{He}^{*}+\mathrm{He}$ could entirely explain experimental observations [50,73,86-92].

The $\mathrm{He}_{2}{ }^{+}+\mathrm{e}^{-} \rightarrow \mathrm{He}^{*}+\mathrm{He}$ dissociation process has been investigated by Cohen [93] and Guberman [94]. The diabatic potential energy curves dissociating into the atomic $3 s^{1} \mathrm{~S}$ and $3 s^{3} \mathrm{~S}$ states cross the potential energy curve of vibrationally excited $\mathrm{He}_{2}{ }^{+}$[93]. In a simple picture, one would expect an intensity ratio of 3:1 dictated by the statistical weights of the atomic triplet and singlet states.

The fact that we observe a much stronger population of the triplet levels indicates that this simple picture does not fully account for the high densities and low temperatures
Table 3. Linewidth coefficients for singlet atomic lines in the gas and the liquid phases obtained from fitting the function $\Delta \omega(p)=\frac{\partial \Delta \omega}{\partial p} p+\Delta \omega_{0}$

\begin{tabular}{llll}
\hline$T[\mathrm{~K}]$ & & $\begin{array}{l}\text { Slope, } \frac{\partial \Delta \omega}{\partial p} \\
{[\mathrm{~nm} / \mathrm{bar}]}\end{array}$ & $\begin{array}{l}\text { Intercept, } \Delta \omega_{0} \\
{[\mathrm{~nm}]}\end{array}$ \\
\hline 4.0 & Gas & $0.87 \pm 0.11$ & $0.10 \pm 0.10$ \\
4.5 & Gas & $0.70 \pm 0.30$ & $0.16 \pm 0.20$ \\
5.0 & Gas & $0.71 \pm 0.03$ & $0.14 \pm 0.03$ \\
& & & \\
3.8 & Liquid & $0.44 \pm 0.02$ & $0.56 \pm 0.10$ \\
4.0 & Liquid & $0.50 \pm 0.06$ & $0.52 \pm 0.20$ \\
4.5 & Liquid & $0.51 \pm 0.04$ & $0.45 \pm 0.12$ \\
5.0 & Liquid & $0.67 \pm 0.21$ & $0.01 \pm 1.00$ \\
\hline
\end{tabular}

Table 4. Linewidth coefficients for triplet atomic lines in the gas and the liquid phases.

\begin{tabular}{llll}
\hline$T[\mathrm{~K}]$ & & $\begin{array}{l}\text { Slope, } \frac{\partial \Delta \omega}{\partial p} \\
{[\mathrm{~nm} / \mathrm{bar}]}\end{array}$ & $\begin{array}{l}\text { Intercept, } \Delta \omega_{0} \\
{[\mathrm{~nm}]}\end{array}$ \\
\hline 4.0 & Gas & $-0.04 \pm 0.10$ & $0.41 \pm 0.10$ \\
4.5 & Gas & $-0.12 \pm 0.10$ & $0.45 \pm 0.04$ \\
5.0 & Gas & $0.13 \pm 0.04$ & $0.33 \pm 0.04$ \\
& & & \\
3.8 & Liquid & $0.28 \pm 0.01$ & $0.15 \pm 0.02$ \\
4.0 & Liquid & $0.29 \pm 0.01$ & $0.16 \pm 0.02$ \\
4.5 & Liquid & $0.30 \pm 0.01$ & $0.10 \pm 0.02$ \\
5.0 & Liquid & $0.31 \pm 0.02$ & $0.24 \pm 0.10$ \\
\hline
\end{tabular}

present in our cell. Glow discharges at pressures higher than a few millibars are characterised by a high concentration of $\mathrm{He}_{2}{ }^{+}$[95]. Studies under such conditions give also evidence of more complicated reactions involving, for example, three-body collisions, $\mathrm{He}_{3}{ }^{+}$and $\mathrm{He}_{4}{ }^{+}$[95-97].

The observed strong increase of the triplet-singlet conversion rate with pressure in our work could therefore have a number of explanations. Also, the number of data points in the gas phase is limited and, while a linear dependence on pressure is apparent, we cannot rule out higher-order contributions to the slopes in Figure 11.

In the liquid phase, the triplet-singlet ratio is lower for pressures slightly above SVP than in the gas phase (at slightly below SVP). The ratio, then, increases with pressure, but not as rapidly as in the gas phase. Also, the triplet-singlet ratio depends on the temperature of the isotherm. Higher temperatures show higher singlettriplet conversion rates. Given that the process appears to be more efficient in the gas phase at similar densities, a possible interpretation is that fewer particles are solvated at $5.0 \mathrm{~K}$ than at $3.8 \mathrm{~K}$. At higher temperatures, the $\mathrm{He}_{2}{ }^{+}, \mathrm{He}_{3}{ }^{+}$and $\mathrm{He}_{4}{ }^{+}$in gas bubbles would have a greater chance to recombine with electrons than at lower temperatures because solvated ions and electrons have lower mobility in liquid helium [98-101].

In conclusion, we have demonstrated a new design for the efficient acquisition of fluorescence spectra of helium excited by corona discharge over a wide range of pressures at cryogenic temperatures covering the gas and liquid phases. Several thousand spectra were recorded, including in the previously unexplored region of dense 


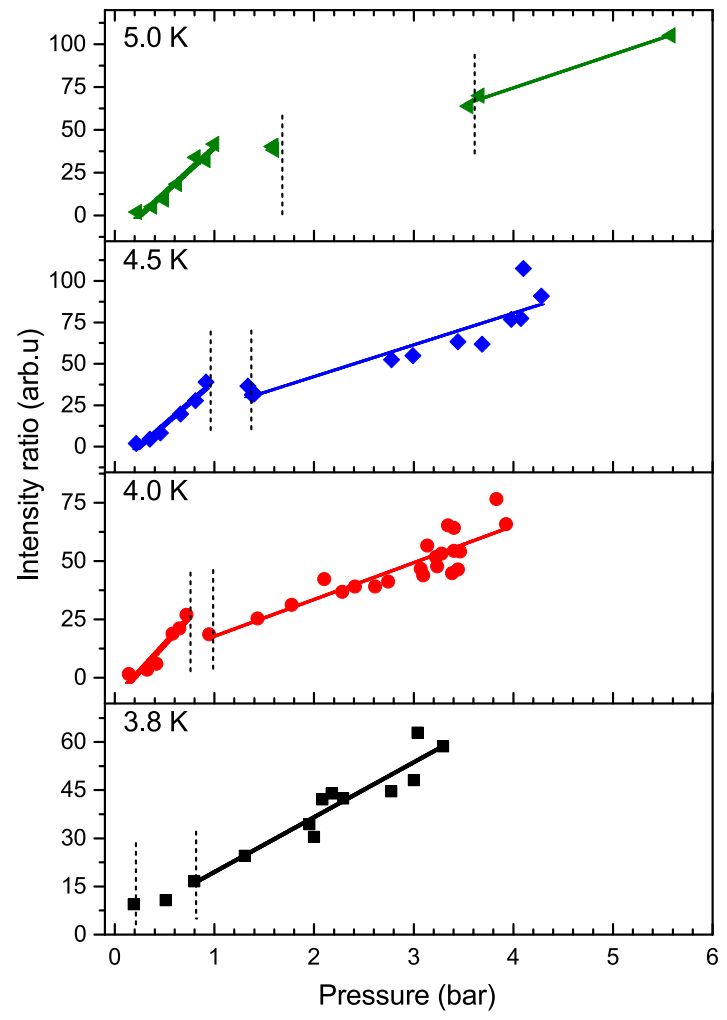

Fig. 11. Pressure dependence of the intensity ratio of triplet $\left(3 s^{3} S_{0} \rightarrow 2 p^{3} P_{0,1,2}\right)$ and singlet $\left(3 s^{1} S_{0} \rightarrow 2 p^{1} P_{1}\right)$ lines at 3.8 , $4.0,4.5$ and $5.0 \mathrm{~K}$. The straight lines indicate regions that show linear dependence on pressure. The dashed lines mark the boundaries of the crossover regions.

cryogenic helium gas, and the atomic fluorescence lines emerging from the $n=3$ singlet and triplet states were analysed. The dependence of lineshifts and linewidth on pressure supported the supposition that a significant number of atoms are solvated in the liquid phase. In the gas phase no appreciable dependence on the cell temperature was observed, indicating that plasma properties dictated the mechanisms of the excitation, energy transfer and emission processes. The fluorescence from triplet levels dominated the singlet levels by an intensity ratio of up to two orders of magnitude. This exceptionally high conversion rate was attributed to vibrationally excited molecular ion precursors undergoing dissociative recombination.

The demonstration of a new cryogenic high pressure discharge cell is encouraging for the future exploration of molecular interactions in liquid helium. The high throughput by which fluorescence light is collected and measured opens new avenues for investigating a large region in the $P, T$ parameter space, including in the gas, supercritical, liquid and superfluid phases, providing new insight into solvation phenomena at the nanoscale. The methodological advance demonstrated in this work will be also important for the measurement of trace impurities in gases and liquids by fluorescence spectroscopy, using corona discharges for electronic excitation.

$\mathrm{KvH}$ acknowledges funding by the British Council (Alliance Programme). $\mathrm{KvH}$ is grateful for funding by The Leverhulme
Trust (Res. Grant F00212AH), the Royal Society (Int. Exchange Grant IE130173), COST action CM 1405 (Molim). LGML acknowledges financial support from ERASMUS and the Mexican Consejo Nacional de Ciencia y Tecnología (CONACYT) Scholarship No. 310668, ID 215334, NS acknowledges financial support Iraqi Minitry of Higher Education and Scientific Research (MoHESR) Scholarship No. 3312/20141252. We thank N. Bonifaci, CNRS, Grenoble, for advice in the design and being introduced to corona discharges. We are indebted to the Space Research Centre at the University of Leicester who kindly lent us the Oxford Instr. closed cycle cryostat (M. Sims, D. Ross, J. Williams, J. Lapington, R. Limpenny).

Open Access This is an open access article distributed under the terms of the Creative Commons Attribution License (http://creativecommons.org/licenses/by/4.0), which permits unrestricted use, distribution, and reproduction in any medium, provided the original work is properly cited.

\section{References}

1. W.S. Dennis, E. Durbin Jr., W.A. Fitzsimmons, O. Heybey, G.K. Walters, Phys. Rev. Lett. 23, 1083 (1969)

2. K. von Haeften, T. Laarmann, H. Wabnitz, T. Möller, Phys. Rev. Lett. 87, 153403 (2001)

3. F. Vigliotti, M. Chergui, M. Dickgiesser, N. Schwentner, Faraday Discuss. 108, 139 (1997)

4. P. Moroshkin, A. Hofer, A. Weis, Phys. Rep. 469, 1 (2008)

5. M. Mudrich, F. Stienkemeier, Int. Rev. Phys. Chem. 33, 301 (2014)

6. M.P. Ziemkiewicz, D.M. Neumark, O. Gessner, Int. Rev. Phys. Chem. 34, 239 (2015)

7. K. von Haeften, T. Laarmann, H. Wabnitz, T. Möller, Phys. Rev. Lett. 88, 233401 (2002)

8. H. Gharbi Tarchouna, N. Bonifaci, F. Aitken, L.G. Mendoza-Luna, K. von Haeften, J. Phys. Chem. Lett. 6, 3036 (2015)

9. K. von Haeften, K. Fink, Eur. Phys. J. D: At. Mol. Opt. Plasma Phys. 43, 121 (2007)

10. K.D. Closser, M. Head-Gordon, J. Phys. Chem. A 114, 8023 (2010)

11. K. von Haeften, T. Laarmann, H. Wabnitz, T. Möller, K. Fink, J. Phys. Chem. A 25, 7316 (2011)

12. K.D. Closser, O. Gessner, M. Head-Gordon, J. Chem. Phys. 140, 134306 (2014)

13. K.D. Closser, Q. Ge, Y. Mao, Y. Shao, M. Head-Gordon, J. Chem. Theory Comput. 11, 5791 (2015)

14. M. Dehdashti-Jahromi, H. Farrokhpour, J. Phys. Chem. C 119, 18641 (2015)

15. H. Farrokhpour, M. Dehdashti-Jahromi, J. Mol. Liq. 230, 190 (2017)

16. C.M. Surko, F. Reif, Phys. Rev. 175, 229 (1968)

17. C.M. Surko, F. Reif, Phys. Rev. Lett. 20, 582 (1968)

18. C.M. Surko, G.J. Dick, F. Reif, W.C. Walker, Phys. Rev. Lett. 23, 842 (1969)

19. C.M. Surko, R.E. Packard, G.J. Dick, F. Reif, Phys. Rev. Lett. 24, 657 (1970) 
20. W.G. Rellergert, S.B. Cahn, A. Garvan, J.C. Hanson, W.H. Lippincott, J.A. Nikkel, D.N. McKinsey, Phys. Rev. Lett. 100, 025301 (2008)

21. T.M. Ito, S.M. Clayton, J. Ramsey, M. Karcz, C.-Y. Liu, J.C. Long, T.G. Reddy, G.M. Seidel, Phys. Rev. A 85, 042718 (2012)

22. R.E. Packard, F. Reif, C.M. Surko, Phys. Rev. Lett. 25, 1435 (1970)

23. M. Stockton, J.W. Keto, W.A. Fitzsimmons, Phys. Rev. Lett. 24, 654 (1970)

24. J.C. Hill, O. Heybey, G.K. Walters, Phys. Rev. Lett. 26, 1213 (1971)

25. M. Stockton, J.W. Keto, W.A. Fitzsimmons, Phys. Rev. A 5, 372 (1972)

26. J.W. Keto, M. Stockton, W.A. Fitzsimmons, Phys. Rev. Lett. 28, 792 (1972)

27. J.W. Keto, F.J. Soley, M. Stockton, W.A. Fitzsimmons, Phys. Rev. A 10, 872 (1974)

28. J.W. Keto, F.J. Soley, M. Stockton, W.A. Fitzsimmons, Phys. Rev. A 10, 887 (1974)

29. F.J. Soley, W.A. Fitzsimmons, Phys. Rev. Lett. 32, 988 (1974)

30. D.W. Tokaryk, R.L. Brooks, J.L. Hunt, Phys. Rev. A 48, 364 (1993)

31. A. Trottier, A.I. Jirasek, H.F. Tiedje, R.L. Brooks, Phys. Rev. A 61, 52504 (2000)

32. S.G. Kafanov, A.Y. Parshin, I.A. Todoshchenko, J. Exp. Theor. Phys. 91, 991 (2000)

33. A.Y. Parshin, I.A. Todoshchenko, S.G. Kafanov, Physica B 284, 91 (2000)

34. Z. Li, N. Bonifaci, A. Denat, V.M. Atrazhev, IEEE Trans. Dielectr. Electr. Insul. 13, 624 (2006)

35. Z. Li, N. Bonifaci, F. Aitken, A. Denat, K. von Haeften, V. Atrazhev, V.A. Shakhathov, IEEE Trans. Dielectr. Electr. Insul. 16, 742 (2009)

36. Z.-L. Li, N. Bonifaci, F. Aitken, A. Denat, K. von Haeften, V.M. Atrazhev, V.A. Shakhatov, Eur. Phys. J. Appl. Phys. 47, 22821 (2009)

37. L.G. Mendoza-Luna, N.M.K. Shiltagh, M.J. Watkins, N. Bonifaci, F. Aitken, K. von Haeften, J. Phys. Chem. Lett. 7, 4666 (2016)

38. W. Guo, J.D. Wright, S.B. Cahn, J.A. Nikkel, D.N. McKinsey, Phys. Rev. Lett. 102, 235301 (2009)

39. W. Guo, S.B. Cahn, J.A. Nikkel, W.F. Vinen, D.N. McKinsey, Phys. Rev. Lett. 105, 045301 (2010)

40. H. Buchenau, J.P. Toennies, J.A. Northby, J. Chem. Phys. 95, 8134 (1991)

41. T. Jiang, C. Kim, J.A. Northby, Phys. Rev. Lett. 71, 700 (1993)

42. B.E. Callicoatt, K. Förde, T. Ruchti, L. Jung, K.C. Janda, J. Chem. Phys. 108, 9371 (1998)

43. S. Yurgenson, C.-C. Hu, C. Kim, J.A. Northby, Eur. Phys. J. D 9, 153 (1999)

44. S. Denifl, M. Stano, A. Stamatovic, P. Scheier, T.D. Märk, J. Chem. Phys. 124, 054320 (2006)

45. H. Schöbel, P. Bartl, C. Leidlmair, S. Denifl, O. Echt, T.D. Märk, P. Scheier, Eur. Phys. J. D 63, 209 (2011)

46. M. Joppien, R. Karnbach, T. Möller, Phys. Rev. Lett. 71, 2654 (1993)

47. M. Joppien, R. Müller, T. Möller, Z. Phys. D: At. Mol. Clust. 26, 175 (1993)

48. R. Karnbach, M. Joppien, T. Möller, J. Chim. Phys. 92, 499 (1995)
49. R. Fröchtenicht, U. Henne, J.P. Toennies, A. Ding, M. Fieber-Erdmann, T. Drewello, J. Chem. Phys. 104, 2548 (1996)

50. K. von Haeften, A.R.B. de Castro, M. Joppien, L. Moussavizadeh, R. von Pietrowski, T. Möller, Phys. Rev. Lett. 78, 4371 (1997)

51. T. Möller, K. von Haeften, T. Laarman, R. von Pietrowski, Eur. J. Phys. D 9, 5 (1999)

52. D.S. Peterka, A. Lindinger, L. Poisson, M. Ahmed, D.M. Neumark, Phys. Rev. Lett. 91, 043401 (2003)

53. K. von Haeften, T. Laarmann, H. Wabnitz, T. Möller, J. Phys. B: At. Mol. Opt. Phys. 38, S373 (2005)

54. D.S. Peterka, J.H. Kim, C.C. Wang, L. Poisson, D.M. Neumark, J. Phys. Chem. A 111, 7449 (2007)

55. L.G. Mendoza-Luna, M. Watkins, K. von Haeften, N. Bonifaci, F. Aitken, Eur. Phys. J. D 67, 122 (2013)

56. D. Buchta, S.R. Krishnan, N.B. Brauer, M. Drabbels, P. O'Keeffe, M. Devetta, M. Di Fraia, C. Callegari, R. Richter, M. Coreno et al., J. Chem. Phys. 139, 084301 (2013)

57. A.C. LaForge, D. Regina, G. Jabbari, K. Gokhberg, N.V. Kryzhevoi, S.R. Krishnan, M. Hess, P. O'Keeffe, A. Ciavardini, K.C. Prince et al., Phys. Rev. A 93, 050502 (2016)

58. O. Kornilov, O. Bünermann, D.J. Haxton, S.R. Leone, D.M. Neumark, O. Gessner, J. Phys. Chem. A 115, 7891 (2011)

59. O. Bünermann, O. Kornilov, D.J. Haxton, S.R. Leone, D.M. Neumark, O. Gessner, J. Chem. Phys. 137, 214302 (2012)

60. O. Bünermann, O. Kornilov, S.R. Leone, D.M. Neumark, O. Gessner, IEEE J. Sel. Top. Quantum Electron. 18, 308 (2012)

61. Y. Ovcharenko, V. Lyamayev, R. Katzy, M. Devetta, A. LaForge, P. O'Keeffe, O Plekan, P. Finetti, M. Di Fraia, M. Mudrich et al., Phys. Rev. Lett. 112, 073401 (2014)

62. A. Heidenreich, B. Grüner, D. Schomas, F. Stienkemeier, S.R. Krishnan, M. Mudrich, J. Mod. Opt. 64, 1061 (2017)

63. N. Bonifaci, F. Aitken, V.M. Atrazhev, S.L. Fiedler, J. Eloranta, Phys. Rev. A 85, 042706 (2012)

64. S.L. Guberman III, W.A. Goddard, Chem. Phys. Lett. 14, 460 (1972)

65. S.L. Guberman, W.A. Goddard III, Phys. Rev. A 12, 1203 (1975)

66. D. Vrinceanu, S. Kotochigova, H.R. Sadeghpour, Phys. Rev. A 69, 022714 (2004)

67. N.F. Allard, B. Deguilhem, F.X. Gadea, N. Bonifaci, A. Denat, Europhys. Lett. 88, 53002 (2009)

68. N.F. Allard, N. Bonifaci, A. Denat, Europhys. Lett. 88, $53002(2009)$

69. N.F. Allard, B. Deguilhem, A. Monari, F.X. Gadéa, J.F. Kielkopf, Astron. Astrophys. 559, A70 (2013)

70. S.L. Fiedler, J. Eloranta, J. Low Temp. Phys. 174, 269 (2014)

71. J.-F. Delpech, J. Boulmer, F. Devos, Phys. Rev. Lett. 39, 1400 (1977)

72. J. Stevefelt, J.M. Pouvesle, A. Bouchoule, J. Chem. Phys. 76, 4006 (1982)

73. D.R. Bates, J. Phys. B: At. Mol. Phys. 17, 2363 (1984)

74. J.F. Su, J.L. Nicol, J. Phys. B: At. Mol. Opt. Phys. 23, 2215 (1990)

75. J.F. Su, J.L. Nicol, J. Phys. B: At. Mol. Opt. Phys. 26, 255 (1993) 
76. P. Moroshkin, V. Lebedev, A. Weis, Phys. Rev. A 87, 022513 (2013)

77. S. Denifl, F. Zappa, I. Mahr, J. Lecointre, M. Probst, T.D. Märk, P. Scheier, Phys. Rev. Lett. 97, 043201 (2006)

78. F. Ferreira da Silva, P. Waldburger, S. Jaksch, A. Mauracher, S. Denifl, O. Echt, T.D. Märk, P. Scheier, Chem. Eur. J. 15, 7101 (2009)

79. E. Coccia, F. Marinetti, E. Bodo, F.A. Gianturco, ChemPhysChem 9, 1323 (2008)

80. E. Coccia, F. Marinetti, E. Bodo, F.A. Gianturco, J. Chem. Phys. 128, 134511 (2008)

81. F. Marinetti, E. Yurtsever, F.A. Gianturco, J. Phys. Chem. A 114, 9725 (2010)

82. Z. Li, N. Bonifaci, A. Denat, V.M. Atrazhev, V.V. Atrazhev, J. Electrostat. 66, 263 (2008)

83. L.G. Mendoza Luna, Ph.D. thesis, University of Leicester, Leicester, UK, 2015, p. 45ff

84. N.F. Allard, J. Phys.: Conf. Ser. 1, 012065 (2012)

85. M.J. Shaw, M.J. Webster, J. Phys. B: At. Mol. Phys. 9, $16(1976)$

86. J.A. Hornbeck, J.P. Molnar, Phys. Rev. 84, 621 (1951)

87. R.B. Kay, R.H. Hughes, Phys. Rev. 154, 61 (1967)

88. J.D. Jobe, R.M. St. John, Phys. Rev. A 5, 295 (1972)
89. H.F. Wellenstein,W.W. Robertson, J. Chem. Phys. 56, 1411 (1972)

90. H.F. Wellenstein, W.W. Robertson, J. Chem. Phys. 56, 1077 (1972)

91. H.F. Wellenstein, W.W. Robertson, J. Chem. Phys. 56, $1072(1972)$

92. B. Dubreuil, A. Catherinot, Phys. Rev. A 21, 188 (1980)

93. J.S. Cohen, Phys. Rev. A 13, 86 (1976)

94. S.L. Guberman, J. Chem. Phys. 78, 1404 (1983)

95. R. Deloche, P. Monchicourt, M. Cheret, F. Lambert, Phys. Rev. A 13, 1140 (1976)

96. M.A. Gusinow, R.A. Gerber, J.B. Gerardo, Phys. Rev. Lett. 25, 1248 (1970)

97. J.F. Delpech, J.C. Gauthier, Phys. Rev. A 6, 1932 (1972)

98. F. Aitken, Z. Li, N. Bonifaci, A. Denat, K. von Haeften, Phys. Chem. 13, 719 (2011)

99. F. Aitken, N. Bonifaci, A. Denat, K. von Haeften, J. Low Temp. Phys. 162, 702 (2011)

100. F. Aitken, N. Bonifaci, L.G. Mendoza Luna, K. von Haeften, Phys. Chem. Chem. Phys. 17, 18535 (2015)

101. F. Aitken, N. Bonifaci, K. Von Haeften, J. Eloranta, J. Chem. Phys. 145, 044105 (2016) 